\title{
Correction to:
}

The Pathophysiology of Myeloma Bone

Disease: Bone

\section{Remodelling and the Role of Osteoclasts}

Rebecca E. Andrews, Andrew D. Chantry, and A. John Ashcroft

Correction to:

Chapter 2 in: E. Zamagni (ed.), Management of Bone Disease and Kidney

Failure in Multiple Myeloma, https://doi.org/10.1007/978-3-030-63662-3_2

The book was inadvertently published with the captions of all the figures within text. The correct presentation is given here.

The updated version of this chapter can be found at https://doi. org/10.1007/978-3-030-63662-3_2 


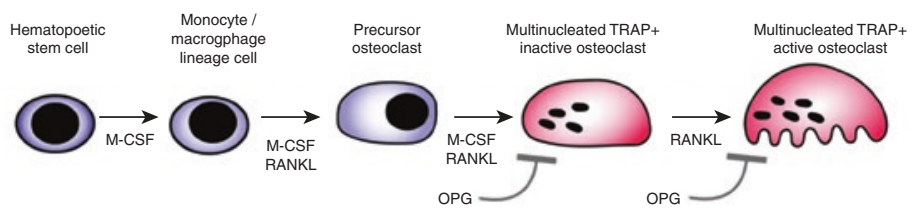

Figure 2.I Osteoclastogenesis. Osteoclasts are derived from hematopoietic stem cells. In the presence of factors such as macrophage colony-stimulating factor (M-CSF) and receptor activator of nuclear factor kappa B ligand (RANKL), precursor OC cells differentiate into multinucleated osteoclast cells, which when activated are capable of bone resorption. Osteoclastogenesis can be inhibited by the production of OPG, which can bind to RANKL, preventing it binding to RANK on the OC cell surface

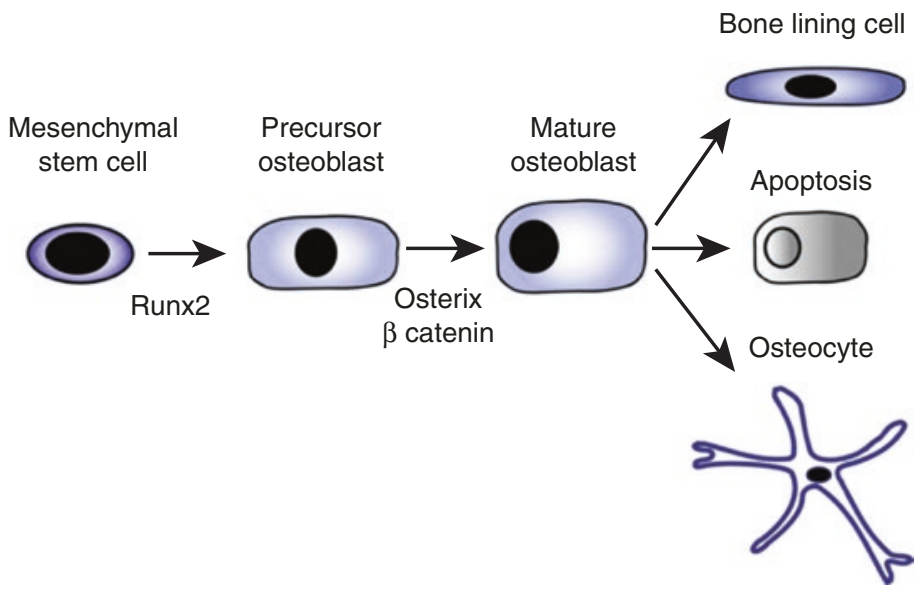

Figure 2.2 Osteoblast differentiation. Osteoblasts are derived from mesenchymal stem cells. Commitment to the osteoblast lineage is regulated by multiple pathways, of which the more established involve Wnt signalling cascades. Mature osteoblasts subsequently either apoptose, become entrapped within the bone matrix to form osteocytes or differentiate into bone-lining cells 


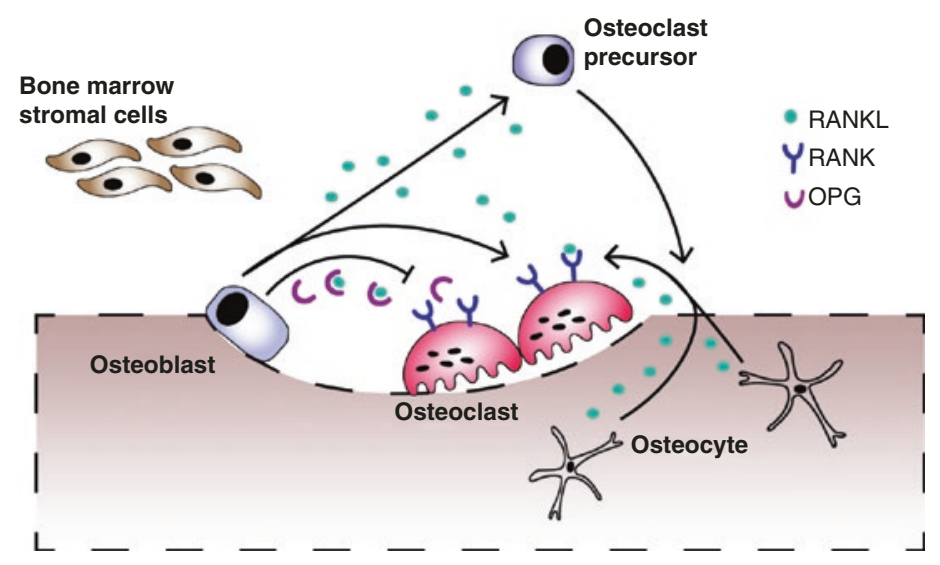

FIGURE 2.3 RANKL/RANK/OPG interaction. There is a close relationship between RANKL and OPG to either drive or antagonise OC differentiation, function and survival. RANKL is produced by both osteocytes and osteoblasts, and when bound to RANK on the surface of osteoclast precursors and immature osteoclasts, differentiation and maturation take place. This signalling pathway is moderated by osteoprotegerin (OPG), a decoy receptor to RANKL which is expressed by osteoblasts, to downregulate OC formation 


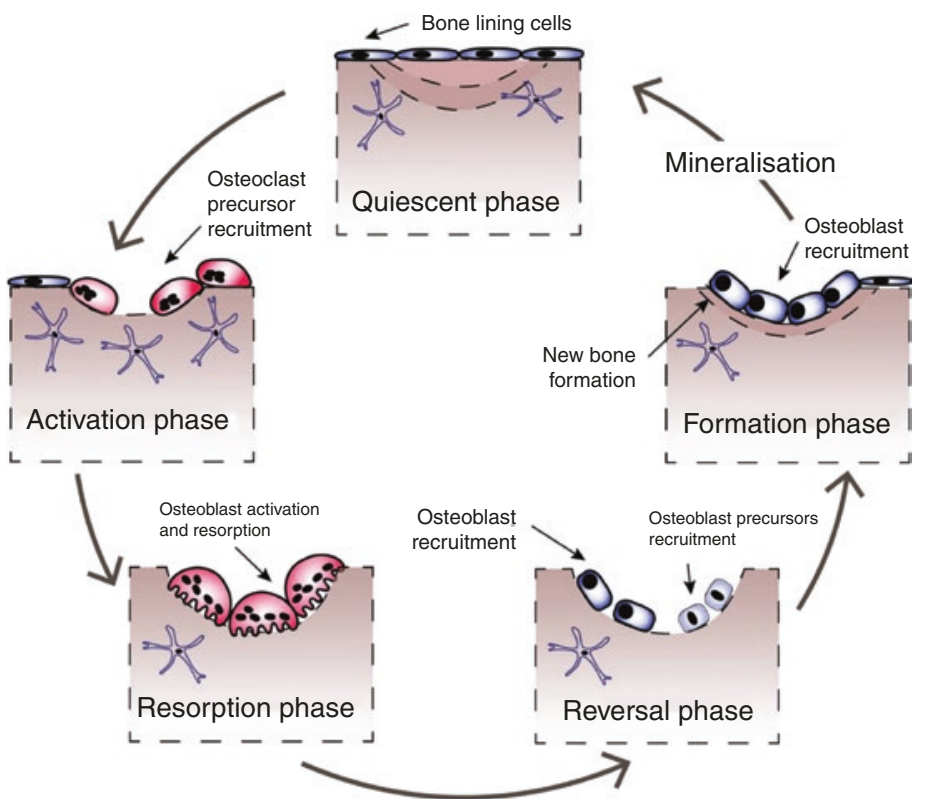

Figure 2.4 Physiological bone remodelling. Bone remodelling involves five distinct phases. Initially, bone microdamage or mechanical loading is recognised by osteocyte cells. Osteocyte-mediated intracellular signalling then triggers an activation phase in which osteoclast precursor cells are recruited to the area of the bone. Subsequent osteoclast maturation, recruitment and bone resorption follow. It is not understood what leads to the transition from resorption to formation, but in this so-called reversal phase, the osteoclasts' role is complete, and osteoblast precursors are recruited. The final active phase is that of bone formation. Osteoblasts lay down osteoid (a new collagen matrix) which will be later mineralised. On completion of the cycle, osteoblast cells either apoptose, become engulfed in the matrix and become osteocytes, or differentiate into bone lining cells that protect the surface of the new bone 


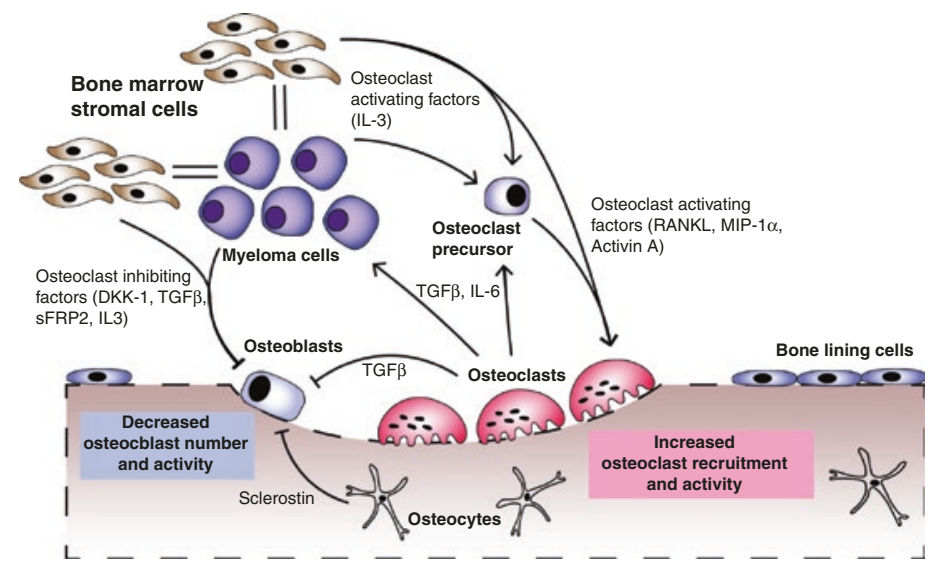

Figure 2.5 Myeloma bone disease: a vicious cycle. The pathophysiology of myeloma bone disease: Cellular interaction between myeloma plasma cells (MPCs) and bone marrow stromal cells (BMSCs) leads to factors that influence the equilibrium of bone turnover. Osteoclast-activating factors (e.g. IL-3, RANKL, MIP-1 $\alpha$ and activin A) are released both from myeloma cells directly and indirect upregulation as a result of adhesion between MPCs and BMSCs. Increased resorption results in increased release of factors such as TGF $\beta$ and IL- 6 from the bone marrow matrix. These promote a positive feedback with upregulation of MPC and osteoclast precursor proliferation, as well as inhibiting osteoblastogenesis. Osteoblast-inhibiting factors (e.g. DKK-1, TGF $\beta$, sFRP2, IL-3) are also released as a result of the presence of MPCs and their interaction with BMSCs. These factors antagonise osteoblast differentiation and function. Sclerostin released from osteocytes is also upregulated in the presence of MPCs, which also inhibits osteoblast function. The overall effect of this is an increase in osteoclast activity and decreased osteoblast activity, resulting in osteolytic bone disease 